\title{
Perspectivas económicas del mundo 2013-2014
}

\section{Luis DELGADILLO P.}

Tomando como referencia el informe semestral del Fondo Monetario Internacional (FMI), se prevé un crecimiento económico global más lento en 2013 y 2014 que el que pronosticó hace tres meses, y luego de las evaluaciones de visualiza una desaceleración en países importantes para economía mundial como China y Brasil y más recesión en Europa. La proyección para el año 2013 sería de 3.1 y para el 2014 fue reducida de 4 a 3,8 por ciento.

En ESTADOS UNIDOS luego de una actualización del presupuesto y de las cifras económicas en la mayor economía del mundo, se espera que el producto bruto interno aumente un $2.0 \%$ este año y un $3.1 \%$ el 2014 , menos que el $2.3 \%$ y $3.2 \%$ de crecimiento proyectado, respectivamente, el 10 de abril en el presupuesto de Obama.

La tasa de desempleo ha mostrado una cierta mejora en los últimos seis meses, pero sigue siendo obstinadamente elevada, con más de un $7 \%$, algo que el Gobierno de Obama dice que se debe al efecto persistente de la peor recesión desde la denominada Gran Depresión de los años 1930.

Según la información facilitada por los economistas de la OMC el 10 de abril de 2013, el ritmo de crecimiento del comercio mundial bajó a 2\% en 2012 en comparación con 5,2\% en 2011, y se prevé que siga siendo lento, de alrededor de 3,3\%, en 2013, mientras la desaceleración económica en Europa continúa reduciendo la demanda mundial de importaciones.

La abrupta desaceleración del comercio en 2012 se ha atribuido al lento crecimiento de las economías desarrolladas y los repetidos episodios de incertidumbre acerca del futuro del euro. La escasa producción y el elevado desempleo en los países desarrollados redujo las importaciones y el ritmo de crecimiento de las exportaciones en las economías desarrolladas y en desarrollo.

La mejora de las perspectivas económicas de los Estados Unidos en 2013 sólo compensaría parcialmente la debilidad continuada de la Unión Europea, 
cuya economía, según estimaciones generalmente aceptadas, se mantendrá estacionaria o incluso se contraerá ligeramente durante el año.

El crecimiento de China debería seguir siendo más rápido que el de otras economías importantes, amortiguando así las repercusiones de la desaceleración, pero las exportaciones seguirán limitadas por la escasa demanda en Europa. El año 2013 parece llamado a ser así casi una repetición de 2012, con una lenta expansión del comercio y la producción, por debajo de sus promedios a largo plazo.

"Los acontecimientos de 2012 deben servir para recordar que las deficiencias estructurales de las economías puestas de manifiesto por la crisis económica no se han subsanado por completo, a pesar de los importantes progresos realizados en algunos sectores. "Los intentos de las economías desarrolladas de establecer un equilibrio entre el crecimiento a corto plazo y las limitaciones fiscales cada vez más estrictas han dado resultados desiguales hasta la fecha, y se ha hecho patente la dificultad de lograr una combinación adecuada de políticas. De modo similar, sigue sin estar claro hasta qué punto han avanzado las economías en desarrollo en la reducción de su dependencia de la demanda externa.

\section{Gráfico 1: Crecimiento del volumen del comercio de mercancías y del PIB mundiales, 2005-2014a}

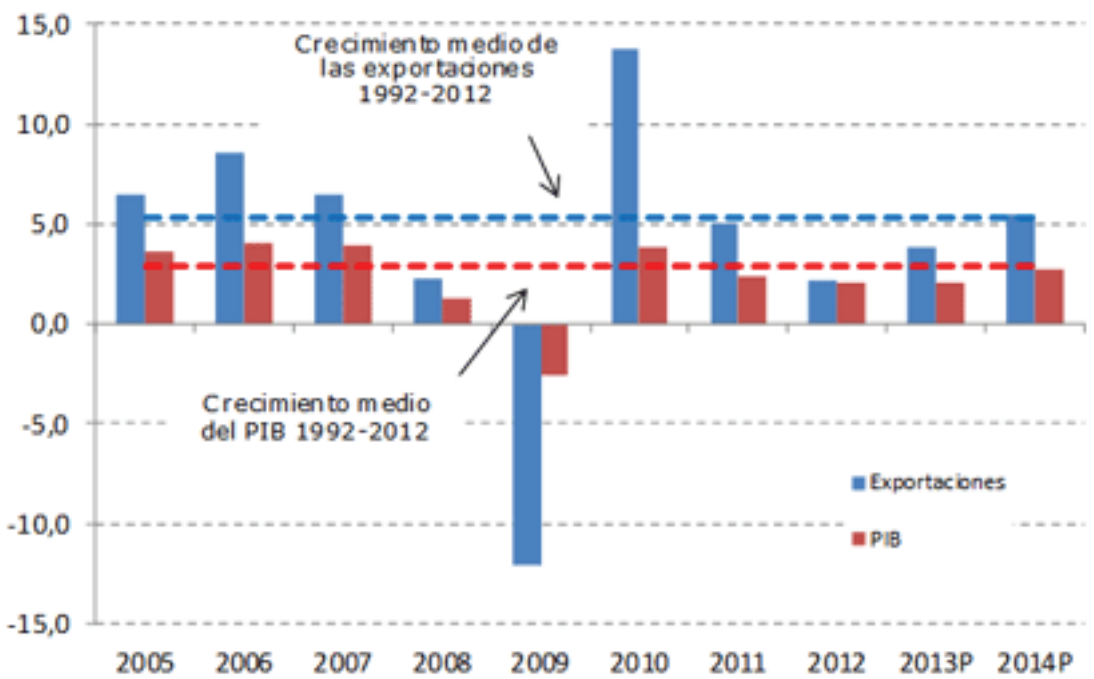

${ }^{a}$ Las cifras correspondientes a 2013 y 2014 son proyecciones.

Fuente: Secretaría de la OMC. 


\section{EL PIB DE JAPÓN TENDRÁ UN CRECIMIENTO DE 2.8\%}

De acuerdo a un reporte de proyecciones económicas de la Oficina del Gabinete en Japón, el PIB de la isla asiática tendrá un crecimiento de $2.8 \%$ en el año fiscal 2013-2014, el cual comenzó en abril pasado; para luego desacelerase fuertemente a $1.0 \%$ en el año fiscal 2014-2015, debido a un aumento planeado al impuesto sobre las ventas. El mencionado impuesto está programado para pasar de 5\% a $8 \%$ a partir del próximo abril, y a $10 \%$ a partir de octubre de 2015.

El aumento al impuesto tendrá un impacto positivo de $0.2 \%$ en el crecimiento del PIB del año fiscal 2013-2014; y un impacto negativo de $0.6 \%$ sobre el crecimiento de la economía en el año fiscal 2014-2015. Se espera que el componente de consumo privado crezca $2.1 \%$ en 2013-2014, y que se desacelere a $0.5 \%$ en $2014-2015$.

\section{EL PRONÓSTICO DE CRECIMIENTO DEL PBI DE CHINA}

Para 2013 fue reducido a 7,8 de 8,1 por ciento en abril. Para 2014, cayó a 7,7 de 8,3 por ciento. La desaceleración de la economía china durante el primer trimestre de 2013, con un crecimiento del PIB del 7,7\% (frente al 7,8\% de 2012 y al 7,9\% del último trimestre de ese año) ha sido notable. La razón es que la inmensa mayoría de los analistas esperaba una expansión de alrededor del $8 \%$ y confiaba en que China podría contribuir positivamente a la tímida recuperación del crecimiento a nivel global. Como es sabido, el Fondo Monetario Internacional (FMI) pronosticó, en sus previsiones del pasado mes de abril, unas tasas para el conjunto del mundo del 3,3\% en 2013 y del 4,0\% en 2014, frente al 3,2\% registrado en 2012.

\section{LAS CAUSAS DE LA DESACELERACIÓN DE CHINA}

Cabe señalar dos posibles grandes causas de la desaceleración.

- En primer lugar, la decisión estratégica de las autoridades de generar un crecimiento más sostenible en el largo plazo y, por tanto, más estable, en pleno proceso de reorientación de la economía desde una pauta basada en la inversión y las exportaciones a otro modelo sustentado en el consumo interno. Las razones para esa reorientación son poderosas: una economía global menos boyante y un incremento de los costes laborales por el aumento del ingreso por habitante, la mayor urbanización, el envejecimiento demográfico (la fuerza de trabajo, en términos absolutos, ya está cayendo), así como una inversión interna poco productiva y generadora de deuda excesiva, capacidad ociosa, sobreconsumo de recursos y deterioro del medio ambiente. 
- En segundo término, las propias dificultades de transición de un modelo a otro, que hacen inevitable un crecimiento más lento y seguramente más volátil, en relación con una casi constante tasa del 10\% exhibida por China desde los años 80 del siglo pasado. También se ha dado a conocer que la contribución del aumento del sector servicios al incremento del PIB ha sido mayor que el del sector manufacturero en ese trimestre.

- Una tercera causa, la llamada "trampa de la renta media", en virtud de la cual China habría alcanzado un nivel de ingreso por habitante que le impediría seguir creciendo a tasas elevadas, puede ser descartada por las razones que se expondrán más adelante.

Pese a que hay algunos analistas que prevén un crecimiento por debajo del $7 \%$, provocado por el crédito excesivo, la alta deuda de los gobiernos locales y la acumulación de préstamos de dudoso cobro, así como por la trampa de la renta media, todo parece indicar, por ahora, que la expansión se situará este año entre el 7\% y el 8\%, cumpliendo más o menos las previsiones del gobierno.

\section{EL IMPACTO GLOBAL}

Así las cosas, cabe plantearse cuáles serán las consecuencias principales de la desaceleración de la economía china desde el 9\%-10\% al 7\%-8\%.

En primer lugar, China seguirá siendo el motor principal de la economía mundial, pero dependiendo de las políticas económicas y financieras aplicadas por ESTADOS UNIDOS y el bloque EUROPEO, pero lo será en menor medida que en años anteriores, si bien éstos se han distinguido por los problemas en la eurozona y, anteriormente, por la crisis financiera internacional. Según las estadísticas del FMI, China fue responsable, en 2010-2012, del 28\% del crecimiento global (14\% de EEUU, 6\% de Japón y 4\% de la UE). Según las previsiones de abril de 2013, esas cifras pasarán al $26 \%, 21 \%,-0,1 \%$ y 12\%) entre 2013 y 2018. En otras palabras, China continuará ejerciendo de motor principal de la economía mundial, pero pasará a ser ayudado, en buena medida, por EEUU.

En segundo lugar, el menor crecimiento del PIB chino se manifestará en una menor demanda de materias primas energéticas y no energéticas $\mathrm{y}$, especialmente, de metales industriales, así como de productos manufacturados intermedios. Algunos países que hoy exportan mucho a China se resentirán: entre ellos, Angola, Australia, Brasil, Canadá, Chile, Rusia, Sudáfrica y Venezuela, por no hablar de Japón y otros países asiáticos del entorno de China, si bien éstos podrían compensar la pérdida con un incremento de las exportaciones de bienes de consumo final. 
* En tercer lugar, al crecer menos las exportaciones, el incremento de las reservas en divisas se hará más lento y, consiguientemente, las colocaciones en títulos del Tesoro de EEUU aumentarán menos. Tal cosa, si es importante, podría provocar un aumento de los tipos de interés en unos EEUU necesitados de capital extranjero para financiar su déficit presupuestario y su déficit exterior.

* Por último, otra consecuencia importante es que se podrían ver beneficiados los competidores de China en la industria intensiva en mano de obra (Vietnam, Bangladesh, India, México, etc.), siempre que las exportaciones crezcan menos y que el coste laboral siga aumentando de manera significativa. Pero ambas cosas no son seguras: conviene tener presente que China dispone de una mano de obra enorme en sus provincias interiores, lo que podría frenar el aumento de los salarios en las industrias de exportación (especialmente si aumenta la urbanización, como pretende el gobierno) y, por tanto, mantener la competitividad de las ventas al exterior.

\section{PERSPECTIVAS ECONÓMICAS PARA AMÉRICA LATINA PREVÉ EL FMI PARA EL 2013 Y 2014}

En el caso de los países de América Latina y el Caribe, el Fondo habla ahora de un crecimiento del $3,4 \%$ en 2013 , cuatro décimas más que el que tuvieron el pasado año pero tres menos de lo previsto. De ahí repuntaría al 3,9\% en 2014, previsión que mantiene intacta. Para Brasil, la mayor economía de la zona, se proyecta una expansión del 3\% este año y del 4\% para el próximo ejercicio, desde el $0,9 \%$ de 2012 . El repunte es consecuencia del recorte de tipos de interés y de las medidas adoptadas para potenciar la inversión privada.

Argentina, Paraguay y Uruguay fueron los países que sufrieron más en el pasado por el frenazo de Brasil. Ahora el FMI también habla de repunte para ellos. En el caso de la economía paraguaya, se pasa de un crecimiento negativo del 1,2\% en 2012 a un avance del $11 \%$ en 2013, para moderarse después al 4,6\% en 2014. Para Argentina se proyecta un crecimiento del 2,8\% este año y del 3,5\% el que viene. Estas previsiones, en todo caso, se hacen con datos alternativos a los que suministra Buenos Aires. También se hace referencia expresa a los controles del Gobierno argentino sobre las importaciones y otros intercambios con el exterior, lo que afecta al clima de negocio y la inversión.

México crecerá un 3,4\% durante los próximos dos años, un frenazo de medio punto frente a 2012. Chile y Perú observan también una moderación del crecimiento (Entre 6 y 6.5\%) hacia su potencial. Para los países de Centroaméríca, el FMI habla de una expansión económica media superior al $4 \%$ gracias al crecimiento de las exportaciones. Panamá destaca claramente 
del resto. La consolidación fiscal de alguno de estos países está atemperando la demanda.

La excepción en la región de América Latina es Venezuela, como resalta el FMI en su mapa de situación al representar al país con un color rosado. De una expansión del 5,5\% el año pasado, la economía venezolana se enfriará hasta el $0,1 \%$ en 2013, para luego repuntar al 2,3\% en 2014. El estancamiento se atribuye a un recorte del gasto público y a una caída del consumo privado.

El FMI, como viene advirtiendo en sus documentos técnicos, indica que el gran reto de las economías emergentes, lo que vale para las de Latinoamérica, será gestionar los flujos de capital una vez que en los avanzados vuelva la recuperación y suban los tipos de interés. Eso, en principio, forma parte del proceso de reequilibrio global. El problema es la volatilidad.

"Con las perspectivas globales mejorando, el gran reto será reequilibrar las políticas para evitar una sobre estimulación”, señala el informe que se someterá este fin de semana a la cumbre del FMI. En otras palabras, se invita a los países emergentes en general a que apoyen cada vez más la demanda interna y controlen la rápida expansión del crédito, para preservar la salud de la banca.

Latinoamérica no será ajena a la desaceleración y la región ve rebajadas sus previsiones para 2013 en cuatro décimas, hasta el 3 por ciento, y en cinco décimas, hasta el 3,4 por ciento para 2014.

Estos datos mantienen el crecimiento de la región en el entorno del 3 ciento de 2012, especialmente por la tendencias a la baja de Brasil y México.

Por otra parte, la recesión en la eurozona parece ser más profunda que lo esperado y se espera que se contraiga 0,6 por ciento este año, más que el 0,4 por ciento augurado en abril.

\section{FMI; BRASIL DEBE INVERTIR MÁS}

El Fondo Monetario Internacional (FMI) recomendó a Brasil aumentar su potencial de crecimiento con inversiones en infraestructuras, después de que el informe de "Perspectivas Económicas Globales" rebajara las estimaciones de crecimiento para la primera economía sudamericana.

El consejero del departamento de Investigación del FMI Thomas Helblíng dijo ayer en la presentación del informe que las previsiones de que Brasil crezca menos este año se deben a la reducción de la demanda mundial, pero también a que el país ha alcanzado su potencial de crecimiento. 
El FMI indicó que Brasil debe mantener sus objetivos de política fiscal y estimular las inversiones en infraestructuras para elevar sus tasas de crecimiento.

El organismo instó a las economías emergentes que "aumenten su potencial de crecimiento" y, en el caso de Brasil, recomendó un aumento en el "muy bajo ritmo de inversiones", algo que afecta a la capacidad de mantener las mejoras registradas hasta 2012.

\section{CONCLUSIONES:}

La primera es que China, a pesar de haber registrado una desaceleración significativa en el primer trimestre de 2013 (con una tasa del 7,7\%, frente al 7,9\% del último trimestre de 2012), podrá seguramente mantener una tasa de entre el 7,5\% y el $8 \%$ durante todo el año. Las razones principales son que su gobierno puede recurrir a la expansión del crédito, a una política fiscal expansiva y, seguramente también, a un incremento, relativamente elevado, de las exportaciones.

La segunda conclusión es que el país está iniciando la reorientación de su pauta de desarrollo desde un modelo basado en la inversión y las exportaciones a otro sustentado en el consumo interior. Las dificultades de ese tránsito hacen que el crecimiento previsible, desde 2014 en adelante, sea más lento, pero sin bajar del 7\%. Fomentar el consumo privado es una tarea complicada, ya que exige aumentar las inversiones públicas en sanidad, educación y pensiones, obligar a las empresas estatales a repartir beneficios y mejorar la distribución de la renta, que ya es la más desigual de todos los países de Asia. Si China lo consigue sin grandes traumas, se convertirá en un motor menos arriesgado y más estable de la economía global.

La tercera conclusión es que las consecuencias de ese cambio de modelo en la economía mundial serán diversas: entre otras, China será un motor menos potente del crecimiento del producto bruto mundial; su demanda de materias primas energéticas y no energéticas bajará o no crecerá tanto, lo que afectará a un buen número de países; la menor importancia de las exportaciones supondrá una menor acumulación de reservas en divisas $\mathrm{y}$, por tanto, de bonos del Tesoro de EEUU; y los competidores de China en la industria intensiva en mano de obra (Vietnam, Bangladesh, México, etc.) se verán beneficiados.

\section{RECOMENDACIONES AL PERÚ}

En este contexto el Perú debe aplicar políticas orientadas a desarrollar y fortalecer a competividad en el marco empresarial y gubernamental; 
además de mantener as políticas económicas en su cauce actual y debe profundizar las inversiones en infraestructura productiva y especialmente en infraestructura agrícola.

En el aspecto comercial el Perú por gestión gubernamental y empresarial debe consolidar los beneficios de los tratados de libre comercio.

Es importante reorientar los recurso del fisco, destinando el $6.5 \%$ a educación y el $4 \%$ a investigación y desarrollo

D El cambio y modernización del SNIP (sistema nacional de inversión pública) es de necesidad nacional y social, para lograr rápidamente los efectos de los recursos del estado en la población.

De manera específica el sector privado tiene que focalizar sus esfuerzos en desarrollar más capacidades competitivas y su tendencia en el mediano y largo plazo debe estar orientada a la producción con progresivo incremento de valor agregado, es decir transformar los recursos naturales para el consumo nacional y exportación.

\section{FUENTES}

Fondo Monetario Internacional

Banco Mundial

Organización Mundial del Comercio

BCRP 